\title{
Information theory analysis of nonlinear regenerative channels
}

\author{
Mariia Sorokina, Stylianos Sygletos, and Sergei Turitsyn \\ Aston Institute of Photonic Technologies, Aston University, B4 7ET Birmingham, UK \\ Email: m.sorokina@aston.ac.uk
}

\begin{abstract}
In this paper we summarize our recently proposed work on the information theory analysis of nonlinear regenerative channels. We discuss fundamental assumptions in our modelling approach and we show that through proper manipulation of the regenerator properties Shannon capacities higher than the limits of the corresponding linear channels can be achieved.

Index Terms - Shannon theory, channel coding, regeneration.
\end{abstract}

\section{INTRODUCTION}

Understanding the true capacity limits of a transmission channel is immensely important for the efficient design of future communication systems. Although the linear case is now well understood [1] a general theory analyzing the capacity performance of nonlinear channels and define corresponding upper bounds has not been established yet. This is because nonlinearity can manifest itself in multiple ways along the transmission link. It can be destructive, giving rise to impairments that affect the signal quality, or it can be constructive achieving the reshaping the signal waveform and the squeezing of any accumulated noise distortion. The latter scenario can be realized by means of all-optical regenerators. Recent progress in the field has led to a variety of such subsystems, characterized by different transfer functions [2][4], that accordingly define nonlinear channels of different properties. As there is a surge in search of new techniques to increase the information throughput, all-optical regeneration represents a unique solution for improving the transparent length and enabling high capacity networking. Nevertheless, information-theoretic analysis of such channels only recently has started appearing in the scientific literature [5], [6].

Here we extend our previous work in the field [6] by proposing a general method to calculate the Shannon capacity in regenerative channels. We show that we can identify the optimum modulation format for a given nonlinear element as a result of a transfer function analysis, rather than optimizing the mutual information functional. The same mapping technique we can be used as well to manipulate the properties of a nonlinear channel for maximizing its transmitted capacity. An example of a regenerative channel that enables higher information rate than the corresponding linear channel is also presented. This demonstrates the fascinating potentials of nonlinearity engineering in information transmission and changes our view on coding and signal packing problem.

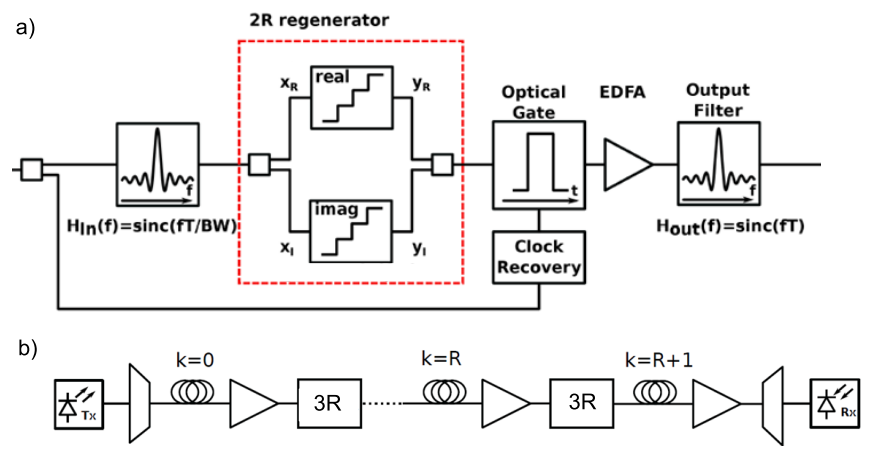

Fig. 1. a) Scheme of $3 R$ regenerator consisting of a $2 R$ regeneration stage, optical sampling and a pulse reformatting filter. Optical sampling is achieved by a switching window of short duration and the NRZ pulse recovery with the use of a matched filter. b) The regenerative channel model where a number of $3 \mathrm{R}$ regenerators are placed equidistantly along the transmission line.

\section{DISCRETE-TIME REGENERATIVE CHANNEL MODEL}

To perform our capacity analysis we made use of a discretetime model, where the non-linear channel was represented by a cascade of noise elements and regenerative transfer functions and the simulations were performed at one sample per symbol. As this approach ignores potential pulse shaping effects in the transmission line, it is important to examine its accuracy against a continuous time model.

The verification has been performed on the specific nonlinear channel example of Fig. 1 (a). It consists of $R$ identical regenerators connected by linear transmission sections of equal length. The signal transmission was distorted by additive white Gaussian noise (AWGN) distributed uniformly along each span $m=1 . . R+1$. To maximize the Shannon capacity the regenerators should be able to suppress both the amplitude and timing distortion of the incoming signal by offering a 3R functionality [7], i.e. re-amplification, re-shaping and retiming. The architecture of such a $3 \mathrm{R}$ subsystem is depicted in Fig.??. At its input a continuous time signal $X_{m}(t)$ experienced the impulse response $h(t)$ of a matched filter which removed the out-of-band noise. Our analysis assumed the use of rectangular pulses, therefore, the matched filter had a sincshaped frequency response, i.e. $H_{\text {in }}(f)=\int_{0}^{T} h(t) e^{-j 2 \pi f t} d t=$ $\operatorname{sinc}(f T) e^{-j 2 \pi f T}$, where $\mathrm{T}$ is the symbol duration. The filtered waveform $Y_{m}(t)$ was subsequently reshaped by the nonlinear element of the regenerator. We have assumed that the two sig- 
nal quadratures were handled independently and experienced the same nonlinear transformation $T(y)$. This enabled to maximize the regenerative efficiency by avoiding any signal-signal interference and to reduce a two-dimensional problem into two independent one-dimensional lattices. Although our method is generic and can be applied to any nonlinear element, here, we have considered without loss of generality, a particular transfer function example : $T(y)=y+a \sin (b y)$, characterized by a large number of regular plateau levels and it can be realized with the scheme proposed in [6].

Optical sampling, followed by signal amplification and linear pulse filtering, complemented the regeneration process with the re-timing functionality. The sampling was performed with an ideal rectangular switching window selecting the central part of each pulse, where the amplitude distortion had been adequately suppressed and there was no influence of timing jitter. The duration of the switching window was a fraction of the symbol period $T$ and it was subject to optimization. After amplifying the signal to compensate the corresponding energy loss, a second matched filter restored the pulses back to their initial shape and duration.

We have simulated the transmission of a continuous time signal through the aforementioned nonlinear channel. The signal was modulated in 16-QAM format using ideal rectangular pulses at a baud rate of $B=28 \mathrm{GBaud} / \mathrm{s}$. In Fig. 2 (a)-(c) the eye-diagram, the waveform and spectrum of the propagating signal after 20 cascaded regenerators have been plotted, and in Fig. 2(d) the ratio of the signal power at the output of each regenerator stage to the power of the launched signal. The results were taken for a switching window which was $2 \%$ of the symbol period $T$. They clearly show that the pulses preserve their initial shape and their average power along the transmission line.

Also, we compared the symbol error rate (SER) performance of the discrete- and continuous- time system models. The SER was calculated by direct error counting using Monte Carlo simulations as a function of optical SNR (OSNR) $O S N R=S N R \frac{B}{2 B_{r e f}}$, where $B_{r e f}=12.5 \mathrm{GHz}$. For the discrete and continuous time signal cases we considered a total number of $2^{25}$ and $5 \cdot 10^{5}$ equiprobable symbols, respectively. The SNR was defined as the ratio of the input signal power $S$ to the noise (zero mean AWGN with variance $N_{m}$ ) added linearly to the signal during transmission at each node $m=$ $1, \ldots, R+1: S N R=S / N$, where accumulated noise is given by $N=\sum N_{m}$, where $N_{m}$ is noise power added after transmission via $m-t h$ node. The calculated SER results are depicted in Fig. 3. Evidently, the effect of noise squeezing is enhanced with the number of regenerators. Furthermore, one can see that for small switching windows using the discrete-timeeffective noise source approximation allows capturing the real behaviour of the regenerative system which is represented by the continuous-time model. However, longer switching window durations affect the shape of the propagating pulses and gives rise to timing jitter phenomena that the discrete-time approach fails to take into account into the SER performance.
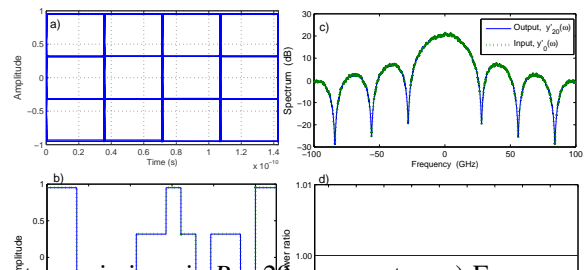

Fig. 2. Waveforms for noiseless transmission via $R=2($ regenerators a) Eyediagram, b) waveform, and c) spectrum of the signd at the output of the last

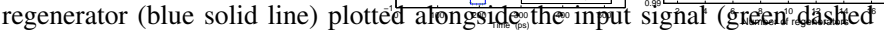
line); d) power ratio of the output signal power at the last regenerator to the power of input signal

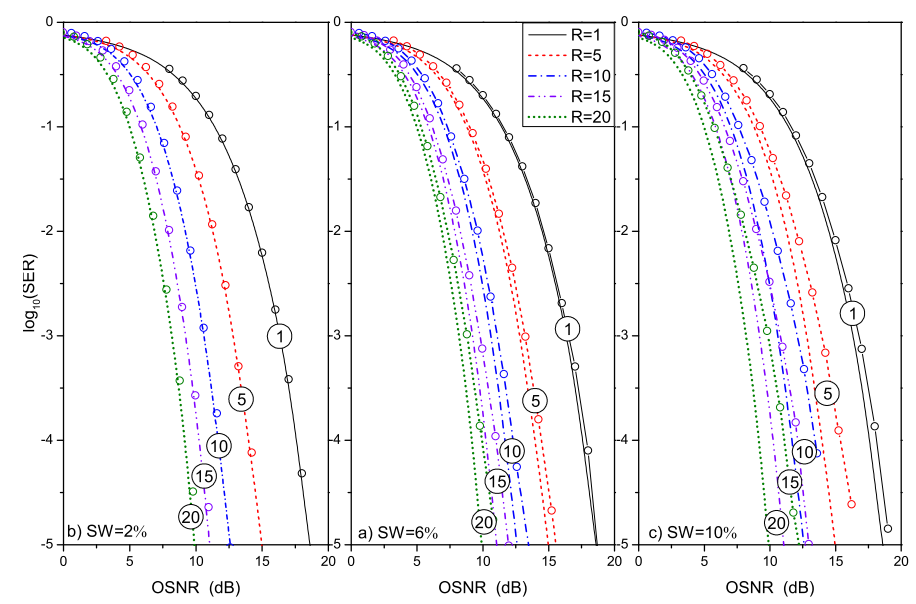

Fig. 3. Comparison of discrete-time and continuous-time modelling SER as a function of OSNR for different values of $S W$ - percentage of the switching window duration with respect to the symbol period. Discrete-time simulations (solid lines) show the upper limit of regenerative continuous-time transmission (shown by lines with circles), which can be achieved with small switching windows. The increase of $S W$ leads to performance degradation. The number of regenerative elements $R$ (with nonlinear TF: $T(y)=y+\pi^{-1} \sin (\pi y)$ ) was varied.

\section{CAPACITY CALCULATIONS}

The definition of the Shannon capacity [1] for an arbitrary memoryless channel involves maximizing the mutual information (MI) functional:

$$
C=\max _{p(x)} \int d x d y p(x) p(y \mid x) \log _{2} \frac{p(y \mid x)}{\int d x p(x) p(y \mid x)},
$$

over all valid input probability distributions $p(x)$ subject to the power constraint $\int d x p(x)|x|^{2} \leq S$. Here statistical properties of the channel are given by the conditional input-output probability density function (pdf) $p(y \mid x)$. The signal evolution is presented by the stochastic map - a discrete version of the Langevin equation for stochastic processes:

$$
Y_{m}=T\left(Y_{m-1}\right)+\eta_{m}, \quad m=1, \ldots, R+1, \quad Y_{0}=x
$$

As we assume regenerators to be placed equidistantly, so for all $m: N_{m}=N_{m+1}=N /(R+1)$, where $N$ is total power of 
noise added to the signal during transmission. The conditional pdf for the output at $m$-th node for each quadrature $y_{m}$ given the input $y_{m-1}$ is found as

$$
p\left(y_{m} \mid y_{m-1}\right)=\frac{1}{\sqrt{2 \pi N_{m}}} \exp \left[-\frac{\left(y_{m}-T\left(y_{m-1}\right)\right)^{2}}{2 N_{m}}\right]
$$

Because of the Markovian property of the process, the conditional pdf of the received signal after propagation through $R$ links, $y_{R+1}$, given the input, $y_{0}=x_{k}$, is expressed by a product of single-step conditional probabilities

$$
p\left(y_{R+1} \mid y_{0}=x_{k}\right)=\int d y_{R} \ldots d y_{1} p\left(y_{R} \mid y_{R-1}\right) \ldots p\left(y_{1} \mid x_{k}\right)
$$

Consequently, the conditional pdf can be expressed through an Onsager-Machlup function or action of the path given by

$$
\mathcal{S}=\Sigma_{m=1}^{R+1} \frac{1}{\sqrt{2 \pi N_{m}}} \frac{\left(y_{m}-T\left(y_{m-1}\right)\right)^{2}}{2 N_{m}}
$$

as follows

$$
p\left(y_{R+1} \mid y_{0}\right)=\int d y_{R} \ldots d y_{1} e^{-\mathcal{S}}
$$

The knowledge of conditional pdf allows one to calculate capacity (or lower bounds) for any regenerative channel by substituting appropriate TF. Further, we consider as example $T(y)=y+a \sin (b y)$.

\section{A. Analysis of conditional pdf}

Thus, we obtain the conditional pdf for the channel output $y_{R+1}$ given input - alphabet point $x_{k}$ :

$p\left(y_{R+1} \mid y_{0}=x_{k}\right)=\int \prod_{m=1}^{R} d y_{m} \frac{1}{\sqrt{2 \pi N_{m}}} e^{-\left(y_{m+1}-y_{m}-a \sin \left(b y_{m}\right)\right)^{2} / 2 N_{m}}$

This expression calculated numerically is plotted in Fig. 4 for the same power of added noise $N=\sum_{m=1}^{R+1} N_{m}$. The conditional pdf demonstrates that the regenerator acts as a quantizer by attracting signal points to the alphabet - one can observe peaks formed around the alphabet points. The alphabet is constructed in accordance with the proposed optimization procedure as a solution of simple equation $T^{\prime}(x)=x$, which for the considered TF defines amplitude distribution $x_{k}=\pi(-K+1+2 k) / b$, with $k=1, \ldots, K$. Note, the regnerative mapping technique allows to identify optimum modulation format without optimization of MI functional.Also, one can see that for the optimal parameters choice, the noise squeezing effect is more pronounced (compare suboptimal case plotted in Fig. 4a with optimal in Fig. 4b), whereas, since suboptimal case peaks are wider, the interference between them is more clear (compare Fig. 4a with inset in Fig. 4b). This stresses the discreteness of the problem: the cell size cannot be arbitrarily small (this is in contrast to the linear channel) and the optimum distribution is discrete. While the noise power $N$ was fixed (here $N=1$ ), placing alphabet points closer to each other (that is, decreasing cell size) resulted in higher interference between the peaks and reduction of the regenerative effect (compare Fig. 4b with tighter packing in Fig. 4c).
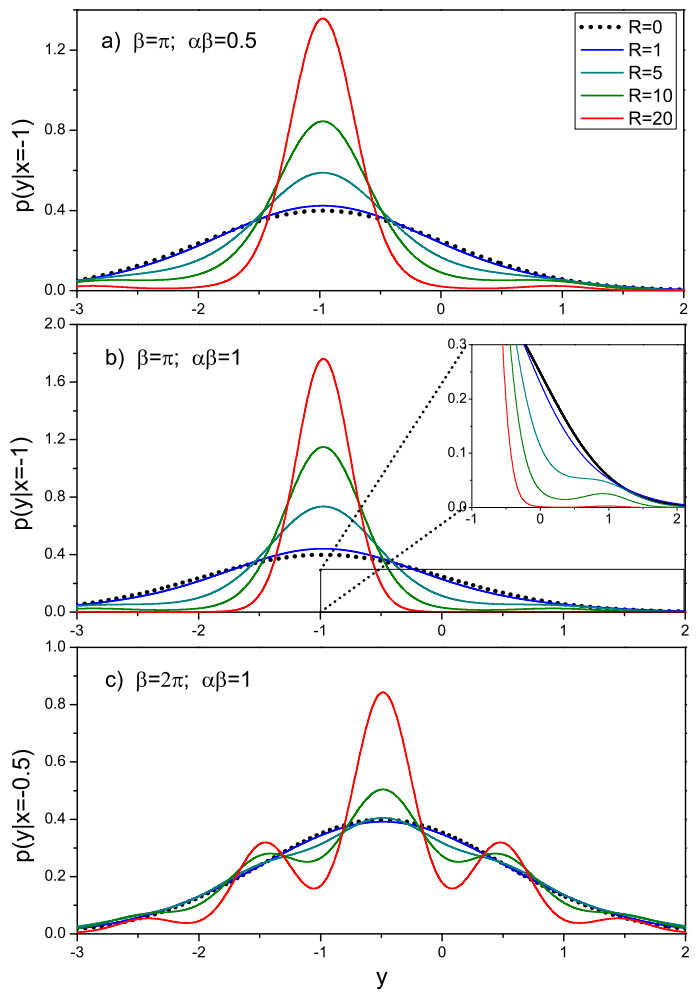

Fig. 4. Conditional pdf $p\left(y=y_{R+1} \mid x\right)$ for a different number of regenerators a) for suboptimal $a b=0.5$ and b) optimal $a b=1$ parameters choice and for the same power of total noise $N=1$. Regenerative transformation results in the formation of peaks around alphabet points given by the regenerative mapping procedure. Increasing the number of regenerators results in more narrow peaks and higher noise squeezing, whereas suboptimal parameters choice, though it results in significant noise reduction, has worse performance than the optimal parameters choice $a b=1$. Decreasing the cell size from $d=2$ (panels a-b) to $d=1$ (panel c) results in worse system performance. It shows that the channel is essentially discrete and the cell size needs to be optimized. Increasing the number of regenerators improves regeneration efficiency.

\section{B. Impact of parameter $b$}

Using conditional pdf, one can calculate the Shannon capacity of the channel with given TF and transformation parameters. Regenerative mapping technique enables finding amplitude distribution optimal for a given regenerative nonlinearity. In considered case, the alphabet is given as $x_{k}=\pi(-K+1+2 k) / b$, where $k=1, \ldots, K$ is an index of an alphabet point. The constraints were $\sum P_{k}\left|x_{k}\right|^{2}=S$ and $\sum P_{k}=1$. The input parameters were: $S N R=S / N$ and $R$. The number of signal points (constellation size $K$ ) was varied until its increase does not affect the capacity calculations by more than $10^{-4}$.

The optimal input probability distribution is found to be a discrete analogue of Gaussian distribution: $P_{k} \sim e^{-x_{k}^{2} / 2 S}$. We differentiate between constrained capacity (MI in Eq. 1 optimized over input probability distribution for a given modulation format, i.e. for fixed values $b$ ) and Shannon capacity (MI in Eq. 1 optimized simultaneously over input probability distribution and modulation format - parameter b).

The Fig. 5 demonstrates impact of parameter $b$ on capacity. 


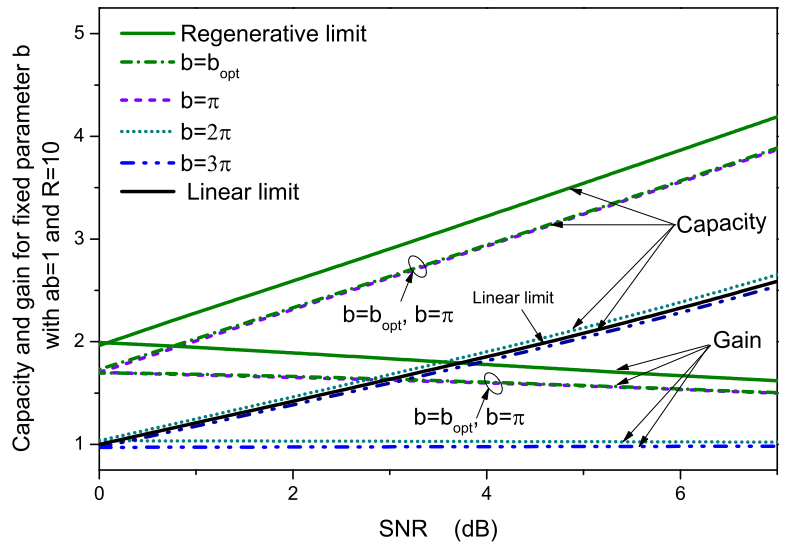

Fig. 5. Constrained capacity of the two-dimensional regenerative channel and the corresponding gain (ratio to the linear Shannon $\operatorname{limit}_{\log _{2}}(1+S N R)$ ) optimized over probability distributions for a given modulation format: equidistant modulation with the cell size $2 \pi / b$ for $b=\pi, 2 \pi, 3 \pi$ for a fixed number of regenerators $R=10$. Shannon capacity of the regenerative channel (optimized over modulation (defined by $b=b_{\text {opt }}$ ) and probability distribution ) and Shannon capacity of a channel with ideal regenerators - regenerative limit are plotted for comparison.

If $b=\pi$, the constrained capacity of regenerative channel is higher than capacity of the corresponding linear channel (linear Shannon limit) and the capacity gain (ratio between two capacities) is higher than 1 . If the cell size is reduced $(=2 \pi / b)$, the constrained capacity becomes smaller, for $b=2 \pi$ the constrained capacity of the channel is approximately equal to the linear Shannon limit and the gain, though still above unity, is roughly equal to one. So, decreasing the cell size below the optimal value, reduces the regenerative effect. Further, decrease of the cell size $b=3 \pi$ degrades the constrained capacity below the linear Shannon limit - regeneration has negative impact: the cell size is so small (compared to the noise variance), that there is high probability that the distorted point will be attracted to the wrong alphabet point and a regenerator will only increase distortion. This demonstrates that the optimal distribution is discrete and the cell size (and, consequently, parameter $b$ ) needs to be optimized.

\section{Impact of parameter a}

Additional parameter $a$ governs the strength of regeneration. In accordance with stability requirement of regenerative mapping technique: $\left|T^{\prime}(x)\right|<1$, it follows that if $a=1 / b$ one can observe plateau formed around the alphabet points; in this case, noise is completely suppressed within the plateaus (this is the best choice of parameter values). When $a=0$ there is no regeneration - the channel converges to linear AWGN channel.

\section{Shannon capacity of the regenerative channel}

Optimizing over parameter $b$ and input probabilities we found maximum of MI over the set of $\left\{P_{k}, b\right\}$ - Shannon capacity of the regenerative channel for $a=1 / b$ see dashdotted lines in Fig.5. The proposed regenerative channel demonstrates that due to nonlinearity it is possible to achieve Shannon capacity higher the Shannon capacity of the linear

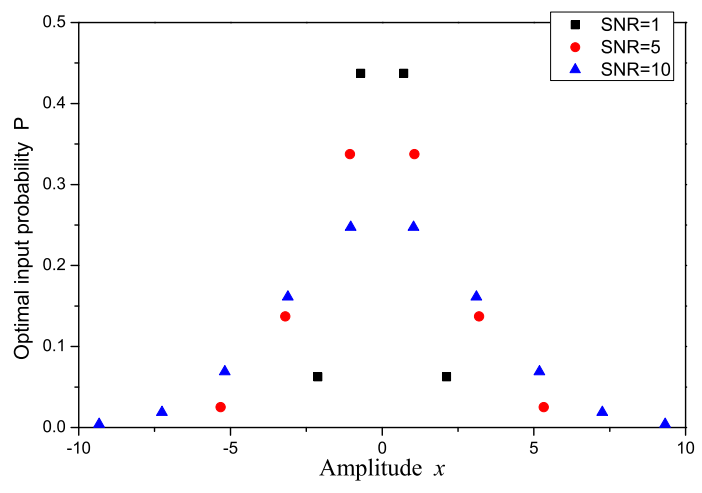

Fig. 6. Optimal input probability

AWGN channel. Here we demonstrate that though TF with plateau is the most efficient, nevertheless, it is not necessary for regeneration. One can see that suboptimal parameter values also provide a capacity increase. The simple analytical equation defines optimization and design rules for optimum modulation format for such nonlinear regenerative channels. Optimal input distribution is plotted in Fig.6, it is discrete with equidistantly placed alphabet points with probability - a discrete analogue of Gaussian distribution.

\section{CONCLusion}

The results illustrate that by suppression of signal distortions, one can achieve Shannon capacity higher than for a system without regenerators operating with the same signal launch power and with the same power of noise added to the signal during transmission.

We are grateful to A.D. Ellis, E. Agrell, and K. Blow for fruitful discussions. and support of the UK EPSRC Programme Grant UNLOC EP/J017582/1.

\section{REFERENCES}

[1] C. E. Shannon, "A Mathematical Theory of Communication," Bell Syst. Tech. J. vol. 27, pp. 379-423, 623-656, 1948.

[2] M. Matsumoto, "Fiber-based all-optical signal regeneration," IEEE J. Sel. Top. Quantum Electron., vol. 18, no. 2, pp. 738752, 2012.

[3] J. Kakande et al., "Multilevel quantization of optical phase in a novel coherent parametric mixer architecture," Nature Photonics, vol. 5, pp. 748-752, 2011.

[4] M Sorokina, 'Design of multilevel amplitude regenerative system,", Optics letters vol. 39 no. 8, pp. 2499-2502 (2014).

[5] K. S. Turitsyn and S. K. Turitsyn, "Nonlinear communication channels with capacity above the linear Shannon limit", Opt. Lett. vol. 37, no. 17, pp. 3600-3602, 2012.

[6] M.A. Sorokina and S.K. Turitsyn, "Regeneration limit of classical Shannon capacity," Nature Communications, vol. 5, Article number: 3861, 2014.

[7] M. Matsumoto "Performance analysis and comparison of optical 3R regenerators utilizing self-phase modulation in fibers", J. Lightw. Technol. vol. 22 , no. 6 , pp.1472-1482 2004 